\title{
YODURIA Y CONCENTRACIÓN DE YODO EN SAL DE CONSUMO EN ESCOLARES PERUANOS DEL NIVEL PRIMARIO
}

\author{
Carolina Tarqui-Mamani1,2,a, Doris Alvarez-Dongo ${ }^{1, b}$, Inés Fernández-Tinco ${ }^{1, c}$
}

\begin{abstract}
RESUMEN
Objetivos. Determinar la yoduria y concentración de yodo sal de consumo en escolares peruanos del nivel primario. Materiales y métodos. Se realizó un estudio transversal. Se incluyó 8023 escolares del nivel primario, que participaron voluntariamente. Se realizó un muestreo probabilístico, multietápico y estratificado, la selección se realizó por muestreo sistemático. La determinación de yoduria se realizó por espectrofotometría (método de Sandell-Kolthoff) y la evaluación de yodo en sal se realizó por volumetría. El procesamiento de datos se realizó mediante análisis para muestras complejas con factor de ponderación. Se calculó medianas, percentiles e intervalos de confianza, se usó la prueba de U Mann Whitney y Kruskall Wallis cuando correspondía. Resultados. A nivel nacional, la mediana de yoduria en los escolares fue $258,53 \mathrm{ug} / \mathrm{L}$, siendo mayor en los varones $(265,90 \mathrm{ug} / \mathrm{L})$ que en las mujeres $(250,77 \mathrm{ug} / \mathrm{L})$. La mediana de yoduria en el área urbana fue mayor $(289,89 \mathrm{ug} / \mathrm{L})$ que en el área rural $(199,67 \mathrm{ug} / \mathrm{L})$, mientras que en los colegios privados fue $315,48 \mathrm{ug} / \mathrm{L}$ y en los públicos fue $241,56 \mathrm{ug} / \mathrm{L}$ ( $p<0,001$ ). La mediana de yodo en sal fue $28,69 \mathrm{mg} / \mathrm{kg}$. El $23,1 \%$ de las muestras de sal, tuvieron menos de $15 \mathrm{mg} / \mathrm{kg}$ de yodo. Conclusiones. La mediana de yoduria en los escolares del nivel primario presenta niveles superiores a las necesarias, según lo recomendado por la OMS, con diferencias entre la zona urbana y rural, y colegios públicos y privados.
\end{abstract}

Palabras clave: Yodo; Deficiencia de yodo; Encuestas nutricionales; Epidemiología, Salud escolar (fuente: DeCS BIREME).

\section{IODURIA AND IODINE CONCENTRATION IN TABLE SALT IN PERUVIAN ELEMENTARY SCHOOLCHILDREN}

\begin{abstract}
Objectives. To determine the ioduria and iodine concentration in table salt in Peruvian elementary schoolchildren. Materials and methods. A cross-sectional study was performed. A total of 8,023 elementary schoolchildren, who voluntarily participated, were included. Multistage stratified probability sampling was performed, and the sample was obtained by systematic selection. loduria was determined via spectrophotometry (Sandell-Kolthoff method), and the amount of iodine in salt was evaluated volumetrically. The data were processed by means of analysis for complex samples with a weighting factor. Medians, percentiles, and confidence intervals were calculated, and the Mann-Whitney $\mathrm{U}$ and Kruskal-Wallis $\mathrm{H}$ tests were used, where appropriate. Results. Nationwide, the median ioduria in schoolchildren was $258.53 \mathrm{ug} / \mathrm{L}$, being higher in boys $(265.90 \mathrm{ug} / \mathrm{L})$ than in girls $(250.77 \mathrm{ug} / \mathrm{L})$. The median ioduria in urban areas was higher $(289.89 \mathrm{ug} / \mathrm{L})$ than that in rural areas $(199.67 \mathrm{ug} / \mathrm{L})$, while it was $315.48 \mathrm{ug} / \mathrm{L}$ in private schools and $241.56 \mathrm{ug} / \mathrm{L}$ in public schools $(p<0.001)$. The median iodine concentration in table salt was $28.69 \mathrm{mg} / \mathrm{kg}$. Of the total salt samples, $23.1 \%$ contained less than $15 \mathrm{mg} / \mathrm{kg}$ of iodine. Conclusions. The median ioduria in elementary schoolchildren exceeded normal levels, according to the criteria of the World Health Organization, with differences between urban and rural areas and public and private schools.
\end{abstract}

Keywords: Iodine; lodine deficiency; Nutritional surveys; Epidemiology, School Health (source: MeSH NLM)

\section{INTRODUCCION}

El yodo es un nutriente que se encuentra mayormente en el mar y en algunos vegetales. Su principal función es la síntesis de las hormonas tiroideas que participan en el desarrollo cerebral y regulación del metabolismo ${ }^{(1)}$. La ingesta deficiente de yodo conduce a presentar manifestaciones clínicas denominadas desórdenes por deficiencia de yodo (DDY) ${ }^{(2)}$. Esta deficiencia es considerada como un problema de salud pública, por ello, la Organización Mundial de la Salud (OMS) y el Fondo de las Naciones Unidas para la Infancia (UNICEF) recomiendan la yodación universal de la sal como una estrategia segura, rentable y sostenible para garantizar el consumo suficiente de yodo por la población ${ }^{(2)}$.

La proporción de hogares que consumen sal adecuadamente yodada ha aumentado considerablemente

\footnotetext{
Centro Nacional de Alimentación y Nutrición. Instituto Nacional de Salud. Lima, Perú

Departamento de Medicina Preventiva y Salud Pública, Facultad de Medicina Humana, Universidad Nacional Mayor de San Marcos. Lima, Perú.

a Doctora en Salud Pública. ${ }^{\mathrm{b}}$ Nutricionista. ${ }^{\mathrm{c}}$ Bióloga

Recibido: 17/08/2016 Aprobado: 23/11/2016
}

Citar como: Tarqui-Mamani C, Alvarez-Dongo D, Fernández-Tinco I. Yoduria y concentración de yodo en sal de consumo en escolares peruanos del nivel primario. Rev Peru Med Exp Salud Publica. 2016;33(4):689-94. doi: 10.17843/rpmesp.2016.334.2552 
en el mundo, llegando a $85 \%$ de los hogares de América Latina y el Caribe; $84 \%$ en los hogares de Asia Oriental y el Pacífico; $72 \%$ en los hogares de Asia occidental y central; $54 \%$ en los hogares de África oriental y meridional, y $51 \%$ en los hogares de Asia meridional (3). El Estado Peruano, ha normado la fortificación de la sal de consumo, con niveles de yodo entre 30 a $40 \mathrm{mg} / \mathrm{kg}^{(4)}$, sin embargo, la OMS indica que la sal de consumo humano debería contener entre 20 a $40 \mathrm{mg} / \mathrm{kg}^{(5)}$.

Según la OMS, la población objetivo de la vigilancia por DDY lo constituyen los escolares, las gestantes y los neonatos ${ }^{(6)}$. Los escolares entre 6 a 12 años son considerados una población vulnerable, al respecto, se ha reportado una mayor prevalencia de bocio en niños cuyas edades fluctúan entre 8 a 10 años. El indicador más utilizado para monitorear la ingesta de yodo es la yoduria, debido a que más del $90 \%$ del yodo consumido se excreta en la orina ${ }^{(6)}$.

En el Perú, los DDY se presentan, principalmente, en los niños y adultos que residen en las zonas rurales, sierra y selva ${ }^{(7,8)}$, en la actualidad, cada vez es menos frecuente encontrar casos de bocio o cretinismo ${ }^{(9)}$; $\sin$ embargo, es más frecuente encontrar medianas de yodo por encima de los niveles necesarios, según lo recomendado por la OMS, tanto en escolares ${ }^{(10,11)}$ como en mujeres peruanas ${ }^{(12,13)}$. Por tal motivo, el objetivo del estudio fue determinar y concentración de yodo en sal de consumo, y la yoduria en escolares peruanos de nivel educativo primario de Perú.

\section{MATERIALES Y MÉTODOS}

Se realizó un estudio observacional y transversal durante el 2013. Se incluyó a escolares matriculados en colegios del nivel primario que voluntariamente aceptaron participar en el estudio, se excluyó a escolares con menstruación, hipertiroidismo y aquellos con medicación a base de yodo o suplementos de hierro. Se realizó un muestreo probabilístico, multietápico y estratificado. Los colegios y escolares fueron seleccionados por muestreo sistemático con arranque aleatorio y con probabilidad proporcional al tamaño de alumnos.

El marco muestral se definió a través de un listado de colegios proporcionado por la Unidad de Estadística del Ministerio de Educación de Perú, que asciende a 37645 colegios (8590 privados y 29055 públicos). La muestra se calculó de manera independiente por dominio y estrato, con un nivel de confianza del 95\%, margen de error que no supera el $6,5 \%$ y una tasa de no respuesta esperada del 15\%. La muestra incluyó 8023 estudiantes de primaria, distribuidos en 719 colegios (públicos y privados) a nivel nacional. Cabe precisar que el diseño, y cálculo del tamaño de muestra fue realizado por el
Instituto Nacional de Estadística e Informática (INEI) en el marco del convenio entre el INEI y el Instituto Nacional de Salud (INS). La planificación de la actividad de vigilancia, y recojo de datos fue realizado por el equipo técnico de la Vigilancia de Indicadores Nutricionales de la Dirección Ejecutiva de Vigilancia Alimentaria y Nutricional del Centro Nacional de Alimentación y Nutrición (CENAN) del INS.

\section{DETERMINACIÓN DE YODURIA}

Se solicitó a los escolares el segundo chorro de la primera micción del día, para ello, se entregó un vaso descartable y un frasco de $30 \mathrm{~mL}$ con tapa rosca, previamente rotulado con el código de identificación del estudiante. La determinación de la concentración de yodo en orina se realizó mediante espectrofotometría que emplea el persulfato de amonio (reacción de Sandell-Kolthoff). Se realizó en los laboratorios de la Dirección Ejecutiva de Ciencia y Tecnología de Alimentos del CENAN, el cual ha sido certificado por los Centros para el Control y la Prevención de Enfermedades (CDC, siglas en inglés). Se consideró a la mediana de yodo urinario menor de $20 \mathrm{ug} / \mathrm{L}$ como severa deficiencia, 20 a $49 \mathrm{ug} / \mathrm{L}$ como moderada deficiencia, 50 a $99 \mathrm{ug} / \mathrm{L}$ como leve deficiencia, 100 a 199 ug/L como niveles de ingesta adecuada; 200 a 299 ug/L como riesgo leve de ingesta de yodo superior a la necesaria y mayor de $300 \mathrm{ug} / \mathrm{L}$ como ingesta de yodo excesiva "riesgo de consecuencias adversas de salud" (5).

\section{DETERMINACIÓN DE YODO EN SAL}

La determinación de la concentración de yodo en sal se realizó mediante el método por volumetría ${ }^{(6)}$ en los laboratorios de la Dirección Ejecutiva de Ciencia y Tecnología de Alimentos del CENAN. Se entregó una bolsa rotulada con cierre hermético a cada estudiante, y se pidió aproximadamente $120 \mathrm{~g}$ de sal de consumo. Se consideró como adecuado el nivel de yodo en sal de consumo cuando se encontró valores $\geq 15 \mathrm{mg} / \mathrm{kg}$ de yodo en sal.

\section{ANÁLISIS DE LOS DATOS}

El procesamiento se realizó mediante análisis para muestras complejas con factor de ponderación en SPSS versión 22. Se calculó medianas y percentiles; además, se utilizó la prueba de Kolmogorv Smirnov para evaluar la normalidad de las variables cuantitativas. Se utilizó pruebas no paramétricas como la $U$ Mann Whitney y Kruskal Wallis para determinar las diferencias en la concentración de yodo en orina y sal según grupos etario, sexo, área, dominio y tipo de colegio, se estableció como nivel de significancia $p<0,05$. Cabe aclarar, que el factor 
de ponderación fue calculado por el INEI. Las variables cuantitativas no tuvieron distribución normal.

\section{ASPECTOS ÉTICOS}

Se solicitó la autorización a la Unidad de Gestión Educativa Local (UGEL), del director del colegio y de los profesores. Asimismo, se solicitó el asentimiento informado por escrito a los escolares de 9 años a más y el respectivo consentimiento informado de los padres del escolar. La identificación de los escolares fue preservada por el equipo técnico y se empleó un código identificador para el análisis de la yoduria en los laboratorios.

El estudio se realizó en el marco de la vigilancia por desórdenes de yodo que, periódicamente, efectúa la Dirección Ejecutiva de Vigilancia Alimentaria y Nutricional del INS, por ello, no se requirió ni fue evaluado por un comité de ética institucional.

\section{RESULTADOS}

Se evalúo a 8023 escolares entre 5 a 17 años, la mayoría de los escolares tuvieron entre 6 y 12 años, la distribución según sexo fue similar entre niños y niñas, la mayoría de los niños residían en el área urbana y el

Tabla 1. Distribución de las características de los escolares del nivel primario. Perú; 2012-2013 ( $n=8023$ )

\begin{tabular}{|cr}
\hline Características & N (\%) \\
\hline Grupo etario (años) & $481(4,9)$ \\
\hline 5 a 6 & $3002(39,1)$ \\
7 a 8 & $2464(29,1)$ \\
\hline 9 a 10 & $1845(23,5)$ \\
\hline 11 a 12 & $231(3,4)$ \\
\hline 13 a 17 & \\
\hline Sexo & $4132(50,9)$ \\
\hline Masculino & $3891(49,1)$ \\
\hline Femenino & \\
\hline Área & $6261(66,5)$ \\
\hline Urbano & $1762(33,5)$ \\
\hline Rural & \\
\hline Dominios & $2347(25,2)$ \\
\hline Lima Metropolitana & $2041(23,1)$ \\
\hline Costa & $2575(35,2)$ \\
\hline Sierra & $1060(16,5)$ \\
\hline Selva & $1451(21,9)$ \\
\hline Tipo de colegio & $6572(78,1)$ \\
\hline Privado & \\
\hline Público & \\
\hline
\end{tabular}

tipo de gestión del colegio fue predominantemente, la administración pública (Tabla 1).

A nivel nacional, la mediana de yoduria fue 258,53 ug/L $\left(P_{25}: 157,15 ; P_{75}: 408,94\right)$ en los escolares, niveles superiores a las necesarias, segun lo recomendado por la OMS (Tabla 2).

Se obtuvieron 7978 muestras de sal de consumo, con una mediana de yodo en sal de $28,69 \mathrm{mg} / \mathrm{kg}\left(\mathrm{P}_{25}: 16,10 ; \mathrm{P}_{75}: 36,77\right)$. El 23,1\% (1674/7978) (IC 95\%: 21,3\%; 25,0\%) de las muestras de sal evaluadas, tuvieron menos de $15 \mathrm{mg}$ de de concentración de yodo (tabla 3 ).

Los departamentos de Moquegua, Madre de Dios, Arequipa, Lambayeque, Tacna y Lima tuvieron niveles

Tabla 2. Distribución de la mediana de yoduria según características de los escolares del nivel primario. Perú; 2012-2013 (n=8023)

\begin{tabular}{|c|c|c|c|c|c|}
\hline Características & $\mathrm{N}=8023$ & $\begin{array}{c}\text { Mediana } \\
\text { (ug/L) }\end{array}$ & $\mathbf{P}_{25}$ & $\mathbf{P}_{75}$ & valor $p$ \\
\hline Grupo etario (años) & & & & & $<0,001^{*}$ \\
\hline 5 a 6 & 481 & 285,6 & 174,1 & 435,5 & \\
\hline 7 a 8 & 3002 & 268,3 & 162,7 & 424,8 & \\
\hline 9 a 10 & 2464 & 257,4 & 158,6 & 400,2 & \\
\hline 11 a 12 & 1845 & 248,0 & 147,9 & 388,7 & \\
\hline 13 a 17 & 231 & 193,4 & 109,9 & 317,7 & \\
\hline Sexo & & & & & $<0,001 \dagger$ \\
\hline Masculino & 4132 & 265,9 & 158,4 & 421,0 & \\
\hline Femenino & 3891 & 250,8 & 155,0 & 397,8 & \\
\hline Área & & & & & $<0,001 \dagger$ \\
\hline Urbano & 6261 & 289,9 & 183,3 & 442,2 & \\
\hline Rural & 1762 & 199,7 & 114,7 & 328,7 & \\
\hline Dominios & & & & & $<0,001^{*}$ \\
\hline $\begin{array}{l}\text { Lima Metropo- } \\
\text { litana }\end{array}$ & 2347 & 311,5 & 209,5 & 453,8 & \\
\hline Costa & 2041 & 287,2 & 171,5 & 446,5 & \\
\hline Sierra & 2575 & 224,0 & 133,3 & 356,6 & \\
\hline Selva & 1060 & 217,2 & 121,7 & 363,4 & \\
\hline Tipo de colegio & & & & & $<0,001 \dagger$ \\
\hline Privado & 1451 & 315,5 & 205,5 & 461,1 & \\
\hline Publico & 6572 & 241,6 & 144,3 & 385,1 & \\
\hline
\end{tabular}

*Prueba de Kruskal Wallis †Prueba U Mann Whitney 
Tabla 3. Distribución de la mediana de yodo en sal según características de los escolares del nivel primario. Perú; 2012-2013 (n=7978)

\begin{tabular}{|c|c|c|c|c|c|}
\hline Características & $N=7978$ & Mediana (mg) & $\mathbf{P}_{25}$ & $P_{75}$ & valor $p$ \\
\hline Grupo etario (años) & & & & & $<0,001^{*}$ \\
\hline 5 a 6 & 478 & 28,8 & 16,3 & 36,2 & \\
\hline 7 a 8 & 2982 & 28,8 & 16,1 & 37,0 & \\
\hline 9 a 10 & 2451 & 28,9 & 16,8 & 36,6 & \\
\hline 11 a 12 & 1838 & 28,7 & 15,5 & 36,9 & \\
\hline 13 a 17 & 229 & 26,5 & 16,8 & 35,2 & \\
\hline Sexo & & & & & $<0,001 \dagger$ \\
\hline Masculino & 4107 & 28,5 & 16,4 & 36,4 & \\
\hline Femenino & 3871 & 28,9 & 15,9 & 37,0 & \\
\hline Área & & & & & $<0,001 \dagger$ \\
\hline Urbano & 6221 & 31,1 & 19,4 & 37,9 & \\
\hline Rural & 1757 & 22,7 & 12,7 & 33,8 & \\
\hline Dominios & & & & & $<0,001^{*}$ \\
\hline Lima Metropolitana & 2331 & 33,4 & 25,5 & 39,5 & \\
\hline Costa & 2019 & 27,4 & 11,7 & 36,5 & \\
\hline Sierra & 2574 & 27,1 & 14,9 & 35,6 & \\
\hline Selva & 1054 & 23,7 & 14,1 & 33,6 & \\
\hline Tipo de colegio & & & & & $<0,001 \dagger$ \\
\hline Privado & 1444 & 33,0 & 23,5 & 38,8 & \\
\hline Publico & 6534 & 27,1 & 14,8 & 35,8 & \\
\hline
\end{tabular}

*Prueba de Kruskal Wallis †Prueba de U Mann Whitney

de yoduria menores de $300 \mathrm{ug} / \mathrm{L}$, mientras que Piura, Cusco, Puno y Amazonas tuvieron niveles adecuados de yoduria (100 a 199 ug/L) (Figura 1).

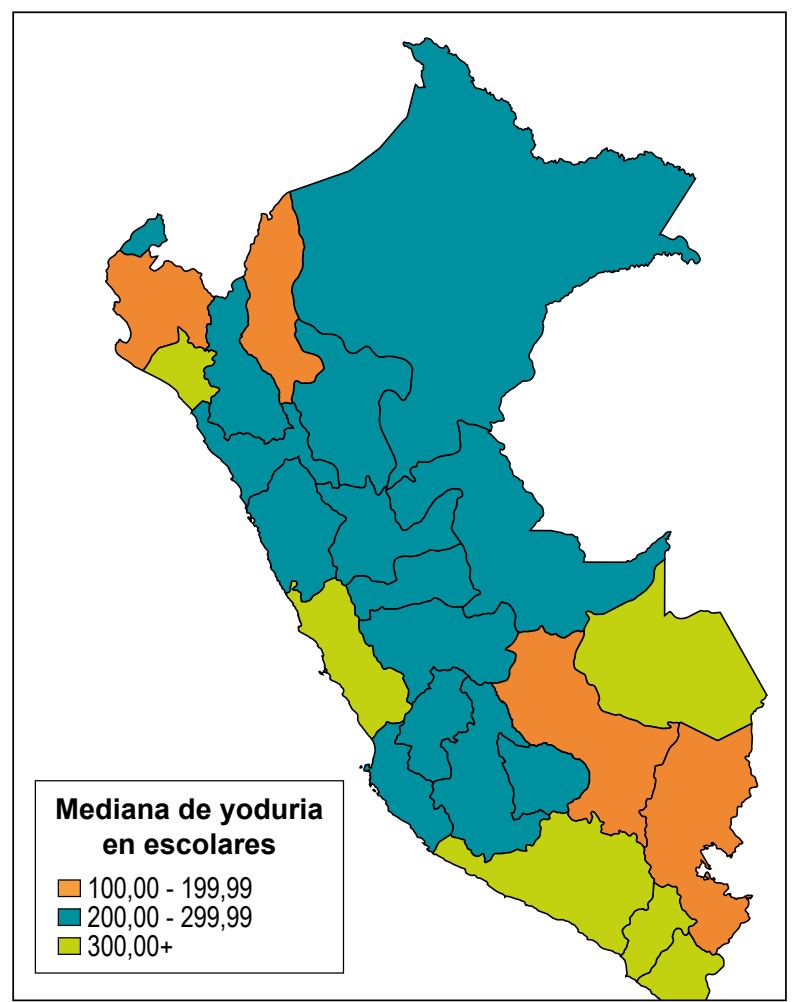

Figura 1. Mediana de yoduria en escolares del nivel primario. Perú; 2012-2013 (ug/L)

\section{DISCUSIÓN}

El Perú, ha sido uno de los países que ha trabajado arduamente en el control del bocio ${ }^{(8)}$, logrando el control sostenido de los desórdenes por deficiencia de yodo a nivel nacional, esto gracias a la intervención del Gobierno Peruano, que dispuso la obligatoriedad de la yodación de la sal de consumo ${ }^{(4)}$. Estudios recientes muestran que la mediana de yoduria presenta valores superiores a los necesarios según lo recomendado por la OMS, tanto en las mujeres (12 a 49 años) ${ }^{(12,13)}$ como en los escolares peruanos de escuelas públicas ${ }^{(10,11)}$.

Los resultados del presente estudio muestran una mediana de yoduria de 258,5 ug/L, considerado por la OMS como valores de ingesta superiores a las necesarias, estos resultados fueron similares a lo reportado por Miranda et al. en escolares peruanos de escuelas públicas (mediana de yoduria: 262,4 ug/L) (11), menor que los escolares venezolanos ${ }^{(1)}$ y similares a los escolares brasileños ${ }^{(14)}$, argentinos ${ }^{(15}$ y colombianos ${ }^{(16)}$. La mediana de yoduria en el área rural estuvo dentro de los niveles considerados adecuados por la OMS, mientras que la mediana de yoduria en el área urbana estuvo por encima de los niveles recomendados por la OMS.

Nuestros hallazgos sugieren que la mediana de yoduria disminuye conforme se incrementa la edad de los escolares, esto difiere de lo reportado por Carvalho et al., que refieren que la mediana de yoduria aumenta con la edad; una 
posible explicación seria que, en Brasil, la concentración de yodo en sal es 20 a 60 mg/kg ${ }^{(17)}$ mientras que, en Perú, la concentración de yodo en sal es 30 a 40 mg/kg.

La mediana de yoduria en Lima Metropolitana presentó niveles mayores a los recomendados por la OMS (311,5ug/L) mientras que la menor concentración de yoduria se presentó en la selva (216,9 ug/L). Estos resultados son contrarios a lo reportado por Miranda et al. (11), que refieren una mediana de yoduria en Lima Metropolitana de 283,3 ug/L y en la selva de 279,7 ug/L. Esto podría deberse a que este estudio se realizó solo en escolares de colegios públicos, a diferencia de nuestra investigación que se realizó con escolares de colegios públicos y privados. Otra razón sería un mayor control del cumplimiento de la fortificación de la sal yodada en Lima Metropolitana y en la costa, esto contribuiría a mejorar el consumo de alimentos elaboradas con sal adecuadamente yodada. También se observó que la mediana de yoduria fue mayor en escolares de colegios privados, es probable que la mejor capacidad adquisitiva de la familia sea un factor que determine la elección y compra de una marca de sal adecuadamente yodada.

Llama la atención, que la mediana de yodo en sal de consumo fue ligeramente menor a lo establecido por la normativa peruana ${ }^{(4)}$, a pesar que la mediana de excreción de yodo en orina está por encima de 200 ug/L; esta situación podría ser explicada por la existencia de otras fuentes de ingesta de yodo como los alimentos procesados o industrializados que agregan aditivos alimenticios como yodato de calcio y yodato de potasio, por lo tanto, la sal no sería la única fuente de yodo sino también existiría otras fuentes de yodo que cubrirían el requerimiento de yodo humano.

La mediana de concentración de yodo en sal en la zona urbana y rural están dentro de los rangos normados en el Perú, siendo mayor en la zona urbana, por otra parte, la menor concentración de yodo en sal fue en la selva $(23,75 \mathrm{ug} / \mathrm{L})$ y sierra $(27,05 \mathrm{ug} / \mathrm{L})$, es posible que en la zona urbana y en la costa peruana exista una mayor facilidad para realizar el monitoreo y control del cumplimiento de la fortificación de la sal.

Es preocupante que la concentración de yodo en sal sea menor a 15 ppm en algunas marcas de sal de consumo, una probable explicación sería la forma de conservación de la sal en el hogar, que podría ser afectada por la humedad ambiental que disminuye la concentración de yodo ${ }^{(18,19)}$, no obstante, también es probable que algunas empresas incumplan la normatividad peruana ${ }^{(4)}$, fortificando la sal con menor cantidad de yodo, o también podría existir sal adulterada cuya marca no corresponde a lo señalado en la bolsa de empaquetamiento.
A juzgar por los resultados del estudio, y según lo recomendado por la OMS ${ }^{(5)}$, algunos sectores poblacionales podrían estar frente a un riesgo de ingesta de yodo superior al necesario. Situación similar se observó en Brasil, que paulatinamente ha disminuido la concentración de yodo en sal hasta llegar a 20 a 60 $\mathrm{mg} / \mathrm{kg}$, sin embargo, no se ha demostrado una relación entre la ingesta de yodo excesiva y aumento de la prevalencia de hipertiroidismo ${ }^{(20)}$.

Por tanto, sería conveniente, reevaluar la pertinencia de mantener los niveles de fortificación de la sal con yodo normados en nuestro país entre 30 a $40 \mathrm{mg} / \mathrm{kg}{ }^{(4)}$ y la posible disminución de los niveles de yodo en sal, entre 20 a $30 \mathrm{mg} / \mathrm{kg}$ de sal, manteniéndonos dentro de los niveles fortificación de yodo en sal recomendado por la OMS (20 a $40 \mathrm{mg} / \mathrm{kg})^{\left({ }^{(6)}\right.}$ y la vigilancia estricta y permanente del cumplimiento de la normatividad peruana sobre la fortificación de la sal con yodo, sobre todo en la sierra y selva peruana. Al respecto, ante una situación similar, el Gobierno de China, dispuso la reducción de la concentración de yodo en sal, de $35 \pm 15 \mathrm{mg} / \mathrm{kg}$ a 20 - $30 \mathrm{mg} / \mathrm{kg}$; disminuyendo la concentración de yodo en orina en escolares de 8 a 10 años de 237,1 ug/L (niveles por encima de lo recomendado por la OMS) a 174,3 ug/L (niveles adecuados según la OMS) ${ }^{(21)}$.

Como limitaciones podemos mencionar que algunas muestras de orina fueron descartadas $(0,5 \%)$, debido a que no se encontraron aptas para el análisis, por dificultades durante el traslado y mantenimiento, sobre todo de zonas afectadas por desastres naturales (inundaciones, huaycos), conflictos sociales internos y variabilidad en el clima; no obstante, el incremento de la muestra frente a la posible tasa de no respuesta permitió contar con una muestra suficiente.

En conclusión, la mediana de yoduria en los escolares peruanos del nivel educativo primario, presenta un valor superior al necesario, según lo recomendado por la OMS, con diferencias significativas entre la zona urbana y rural y entre colegios públicos y privados. Aproximadamente la cuarta parte de las muestras de sal tuvieron niveles por debajo de $15 \mathrm{mg}$ a nivel nacional.

Sugerimos el almacenamiento adecuado de la sal para evitar la pérdida de yodo por humedad. Asimismo, sugerimos reevaluar la pertinencia de mantener los niveles de yodo en sal de consumo normados en nuestro país (30 a $40 \mathrm{mg} / \mathrm{kg}$ ), así como vigilar que la yodación de sal en las plantas de producción se mantenga en niveles adecuados. Es necesaria una vigilancia constante del nivel de yodo en sal de consumo humano de hogares peruanos, y la realización de investigaciones para identificar las potenciales fuentes de ingesta yodo en poblaciones con altos niveles de yoduria. 
Contribuciones de autoría: CTM, ha participado en la concepción del artículo, procesamiento de datos, análisis y redacción del artículo, DAD, IFT participaron en el análisis y redacción del artículo; todos los autores aprobaron la versión final.

Fuente de financiamiento: este estudio fue financiado por el Instituto Nacional de Salud de Perú.
Conflicto de interés: los autores declaran que no existe conflicto de interés en la ejecución o publicación de este estudio.

Agradecimientos: al equipo de supervisión y bioquímica de la Dirección Ejecutiva de Vigilancia Alimentario y Nutricional y la Dirección Ejecutiva de Ciencia y Tecnología de Alimentos del Centro Nacional de Alimentación y Nutrición del INS.

\section{REFERENCIAS BIBLIOGRÁFICAS}

1. Caballero L. Yoduria en escolares y embarazadas del estado Trujillo, Venezuela 2007-2008. Rev. argent. endocrinol. Metab. 2011;28(4):206211

2. Fondo de las Naciones Unidas para la Infancia y Organización Mundial de la Salud, World Summit for Children: Mid-decade goal - Iodine deficiency disorders, informe de la Sesión Especial del Comité Conjunto de UNICEF y la OMS sobre Política de Salud, Ginebra, 27 de enero de 1994.

3. UNICEF. Eliminación sostenible de la carencia de yodo. $1^{\text {a }}$ ed. New York;2008

4. Ministerio de Salud. Programa Nacional de Prevención de deficiencias de micronutrientes (PREDEMI): Normas técnicas para la prevención y control de deficiencias de micronutrientes. Lima: Minsa; $1999.87 \mathrm{p}$.

5. World Health Organization. Salt reduction and iodine fortification strategies in public health [Internet]. Australia: OMS, 2013; [citado 22 Mar 2015]. Disponible en: http://apps.who.int/iris/bitstre am/10665/101509/1/9789241506694_ eng.pdf?ua $=1$

6. World Health Organization (WHO), United Nations Children's Fund (UNICEF), International Council for Control of Iodine Deficiency Disorders (ICCIDD). Assessment of iodine deficiency disorders and monitoring their elimination A guide for programme managers. 3rd ed. WHO/ UNICEF/ICCIDD. Geneva:WHO, 2008

7. Pretell E. Yodo y calidad de vida: eliminación de la deficiencia de yodo en el Perú. Acta Med Per. 2008; 25(4):197-198
8. Pretell E, Higa A. Eliminación sostenida de los desórdenes por deficiencia de yodo en Perú.25 años de experiencia. Acta Med Per. 2008; 25(3):128-134.

9. Organización Mundial de la Salud, Iodine Status Worldwide: WHO global database on iodine deficiency, editado por Bruno de Benoist, et al., OMS, 2004.

10. Instituto Nacional de Salud. Encuesta sobre consumo de sal y yoduria en escolares [monografía en internet]. Lima; INS; 2007 [Citado 20 Jun 2015]. Disponible en: http://www.ins.gob.pe/ insvirtual/BiblioDig/

11. Miranda M, Chávez $H$, Aramburú A, Tarqui-Mamani C. Estado nutricional de yodo en alumnos de escuelas públicas del nivel primario en el Perú, 2009. [Internet]. Lima: Instituto Nacional de Salud;2011. [Citado 23 junio 2015]. Disponible en: http://www.ins.gob. pe/repositorioaps/0/5/zop/zona_izquierda_1/INFORME\%20DDI\%20 2009\%20v2.pdf

12. Higa A, Miranda M, Campos M, Sánchez J. Ingesta de sal yodada en hogares y estado nutricional de yodo en mujeres en edad fértil en Perú, 2008. Rev Peru Med Exp Salud Publica. 2010; 27(2): 195-200

13. Tarqui-Mamani C, Sánchez-Abanto J, Alvarez-Dongo D, Jordán-Lechuga T, Fernández-Tinco I. Concentraciones de yodo en orina y en sal de consumo en mujeres entre 12 a 49 años del Perú. Rev Peru Med Exp Salud Publica. 2015;32(2):252-8.

14. Navarro AM, Oliveira LA, Meirelles CJCS, Costa TMB. Iodação do sal e ingestão excessiva de iodo em crianças. Arch Latinoam Nutr. 2010; $60(4): 355-359$
15. Milani ML, Páez JM, Parco Parisi MA, Parco Parisi L, Pécora R, Bernatené D, et al. Monitoreo de DDI en la provincia de La Rioja (2006). Rev. argent. endocrinol. Metab 2010;47(1):53-7.

16. Gallego M, Loango N, Londoño A, Landazuri P. Niveles de excreción urinaria de yodo en escolares del Quindío, 2006-2007. Rev. salud pública; 2009; 11 (6): 952-960

17. Carvalho AL, Meirelles CJ, Oliveira LA, Costa TM, Navarro AM. Excessive iodine intake in schoolchildren. Eur J Nutr. 2012 Aug;51(5):557-62

18. Biber FZ, Unak P, Yurt F. Stability of iodine content in iodized salt. Isotopes Environ Health Stud. 2002;38(2):8793.

19. Wang GY, Zhou RH, Wang Z, Shi L, Sun M. Effects of storage and cooking on the iodine content in iodized salt and study on monitoring iodine content in iodized salt. Biomed Environ Sci. 1999;12(1):1-9.

20. Camargo RY, Tomimori EK, Neves SC, G S Rubio I, Galrão AL, Knobel M, et al. Thyroid and the environment: exposure to excessive nutritional iodine increases the prevalence of thyroid disorders in Sao Paulo, Brazil. Eur J Endocrinol. 2008;159(3):293-9. doi: 10.1530/EJE-08-0192.

21. Zou Y, Lou X, Ding G, Mo Z, Zhu W, Mao G. Iodine nutritional status after the implementation of the new iodized salt concentration standard in Zhejiang Province, China. BMC Public Health. 2014;14:836.

Correspondencia: Carolina Tarqui-
Mamani
Dirección: Instituto Nacionalde Salud, Calle
Tizóny Bueno 276; Jesús María. Lima, Perú
Correo: carobtm@gmail.com

